\title{
Preexposure to unsignaled food: Autoshaping retardation following differential conditioning of food-tray directed behavior
}

\author{
ANNEMIEKE VAN HEST, FRANS VAN HAAREN, and NANNE E. VAN DE POLL \\ Netherlands Institute for Brain Research, Amsterdam, The Netherlands
}

\begin{abstract}
Preexposure to unsignaled food presentation retards the subsequent acquisition of stimulusdirected responding in autoshaping procedures. We hypothesized that this effect might be a direct function of the extent to which subjects engage in food-tray directed behavior during preexposure. To investigate this notion, unsignaled food presentation was made contingent upon high levels of food-tray directed behavior during preexposure for one group of subjects. For another group of subjects, food presentation during preexposure was contingent upon the occurrence of behavior other than food-tray directed activities. The subjects were then exposed to an autoshaping procedure, in which response-independent food presentations were preceded by the insertion of a response lever into the experimental chamber. Both male and female rats were exposed to these experimental procedures. The subjects that had engaged in high levels of food-tray directed behavior during preexposure exhibited low levels of stimulus-directed behavior during autoshaping, and vice versa. Sex differences were also observed. Males showed more stimulus-directed responding during autoshaping than did females, with no differential effects of preexposure experience.
\end{abstract}

It has previously been shown that preexposure to unsignaled food presentation retards the subsequent acquisition of stimulus-directed behavior in autoshaping procedures (Balsam \& Schwartz, 1981; Downing \& Neuringer, 1976; Engberg, Hansen, Welker, \& Thomas, 1972; Timberlake, 1986; Tomie, 1981). It has also been shown that rats as well as pigeons exhibit high levels of food-tray directed behavior in anticipation of the presentation of response-independent food (Boakes, 1977; Innis, Simmelhag-Grant, \& Staddon, 1983; Timberlake, 1986; van der Schoot, Kop, \& van Haaren, 1987; van Hest, van Haaren, Kop, \& van der Schoot, 1986). Taken together, these observations suggest that the retarded acquisition of stimulus-directed behavior in autoshaping procedures following exposure to response-independent food might be inversely related to the extent to which subjects engage in food-tray directed behavior during preexposure.

The present experiment was designed to investigate whether differential conditioning of food-tray directed behavior during preexposure would result in differences in stimulus-directed behavior during autoshaping. One group of subjects was exposed to a procedure in which the presentation of unsignaled food was explicitly contingent upon the occurrence of food-tray directed behavior. For

This research was conducted while Annemieke van Hest was supported by a grant from the Dutch Organization for the Advancement of Basic Research (NWO 560-258-024) awarded to Frans van Haaren. Frans van Haaren is currently at the Department of Psychology, University of Florida, Gainesville, Florida 32611. Correspondence may be addressed to Annemieke van Hest, Netherlands Institute for Brain Research, Meibergdreef 33, 105 AZ Amsterdam, The Netherlands. another group of subjects, unsignaled food presentations were contingent on the occurrence of behavior other than food-tray directed activities.

Behavioral differences between the sexes have also been observed in different autoshaping procedures (van Haaren, van Hest, \& van de Poll, 1987; van Hest \& van Haaren, 1989). Male rats have been shown to be more likely than females to engage in stimulus-directed behavior when exposed to such procedures. Females, on the other hand, tended to engage more in other than stimulus-directed activities. Male and female Wistar rats thus were chosen for the present experiment to investigate whether or not preexposure conditions would differentially affect the acquisition of stimulus-directed behavior by the two sexes.

\section{METHOD}

\section{Subjects}

Sixteen male and 16 female rats were obtained from Animal House, TNO (Zeist, The Netherlands) when they were 9 weeks old. They were housed in group cages (4 same-sex subjects to a cage) under a reversed light-dark cycle (lights on 3:30 p.m.-3:30 a.m.) upon arrival in the laboratory. The subjects were food deprived and were fed daily to maintain body weights at approximately $80 \%$ of growth-corrected free-feeding body weights. Water was always available in the home cages. The experiment started when subjects were 12 weeks old.

\section{Apparatus}

Experiments took place in eight locally constructed rat chambers which have been described in detail elsewhere (van Haaren et al., 1987).

\section{Procedure}

Adaptation. All subjects were adapted to the experimental chamber for $20 \mathrm{~min}$. Only the houselight was illuminated during this session. Five 45-mg food pellets (Bio-Serve) were placed in the food tray. 
Preexposure. The houselight was illuminated at the beginning of each session, and the levers were retracted from the experimental chamber. For two groups of subjects ( 8 males and 8 females), $45-\mathrm{mg}$ food pellets were presented on a variable interval (VI) 30 -sec schedule of reinforcement (FOODTRAY). Food presentation was contingent upon the first nosepoke in the food tray after the VI had elapsed. Food presentation was accompanied by a 1-sec illumination of the light in the food tray. For the other two groups of subjects ( 8 males and 8 females), food was presented on a chained variable-time (VT) 25-sec differential reinforcement of other behavior (DRO) 5 -sec schedule (OTHER). The DRO contingency insured that at least $5 \mathrm{sec}$ had elapsed between a food-tray entry response and food delivery. Food was presented after the DRO 5-sec interval had elapsed. Food-tray entries during the DRO contingency reset the DRO timer. The subjects were exposed to these experimental conditions for four consecutive sessions, which were terminated after $\mathbf{4 0}$ food pellets had been presented.

Autoshaping. All groups of subjects were then exposed to an autoshaping procedure. The houselight was illuminated at the start of each session. The left lever (CS) was presented on a variable time (VT) 25-sec schedule. CS presentations were accompanied by illumination of the stimulus light located directly above the lever. The stimulus light was extinguished after $5 \mathrm{sec}$ as the lever was retracted from the chamber. Response-independent food (US) was presented immediately upon retraction of the lever from the chamber. All US presentations were accompanied by a 1-sec illumination of the light in the food tray. Subjects were exposed to the autoshaping procedure for five consecutive sessions, which were each terminated after 40 US presentations.

\section{RESULTS}

\section{Preexposure}

Food-tray entry rates (tray entries/min) during the final session of preexposure are shown in Table 1. These rates were log-transformed to increase homogeneity of variance (Winer, 1962) prior to analysis of variance (ANOVA) with the factors sex and condition (FOODTRAY vs. OTHER). Tray-entry rates were higher for males than for females $[F(1,28)=7.83, p<.01]$. The subjects exposed to the FOODTRAY condition showed higher tray-entry rates than subjects exposed to the OTHER condition $[F(1,28)=327.94, p<.01]$. No significant interaction effects were observed.

The mean interval between two successive food presentations was calculated for the different groups of subjects to determine whether the behavioral contingencies during preexposure sessions differentially affected the frequency of food presentation. No differences between subjects exposed to the different experimental contingencies were observed [FOODTRAY: $32.11 \mathrm{sec}$; OTHER: $32.26 \mathrm{sec}, F(1,28)<1.0$, n.s.], nor were differences between the sexes [males: $31.96 \mathrm{sec}$; females: $32.42 \mathrm{sec}$, $F(1,28)<1.0$, n.s.].

\section{Autoshaping}

Tray-entry rates during the autoshaping procedure, as well as the percentage of CS presentations with at least

Table 1

Mean (and Standard Deviation) Tray Entries per Minute During Preexposure

\begin{tabular}{lcc}
\hline & FOODTRAY & OTHER \\
\hline Females & $33.48(8.44)$ & $5.07(2.11)$ \\
Males & $41.59(7.94)$ & $6.31(1.89)$ \\
\hline
\end{tabular}

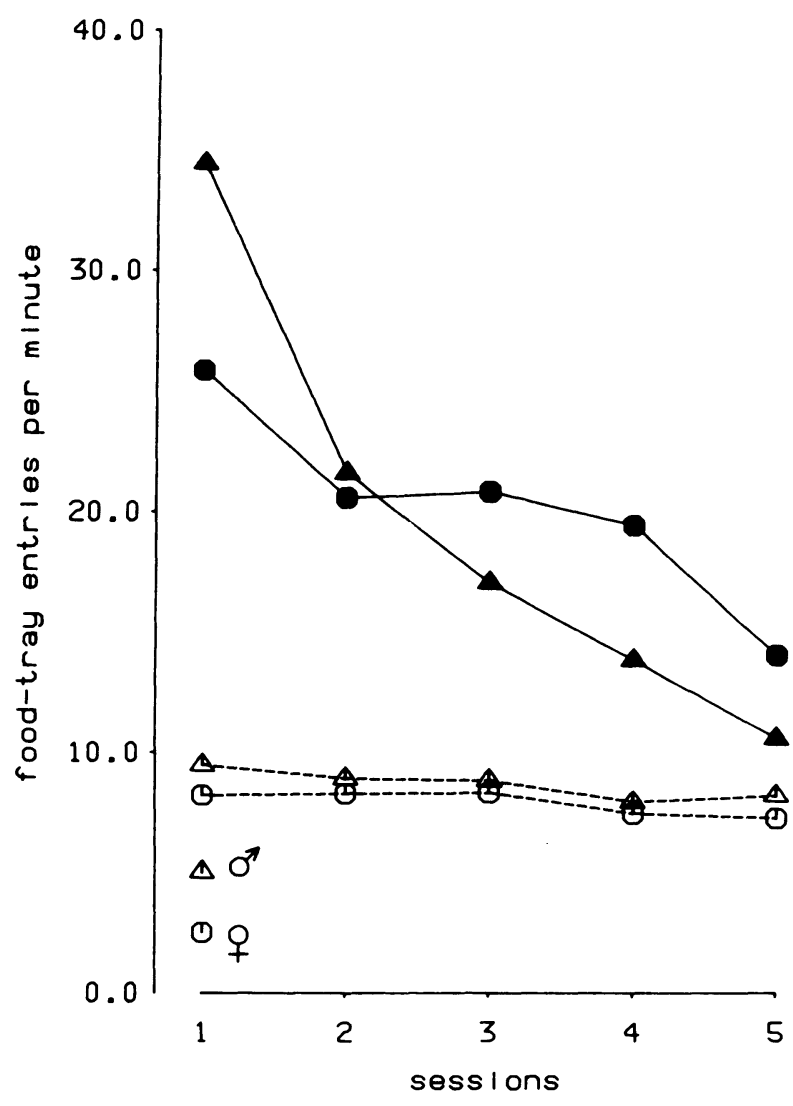

Figure 1. Food tray entries per minute during autoshaping for groups of male (triangles) and female (circles) Wistar rats that were exposed to either the FOODTRAY (solid lines) or OTHER (dotted lines) reinforcement contingencies during preexposure to unsignaled food presentations.

one CS contact, and CS contact rates (CS contacts/min) were analyzed by means of ANOVA with the factors sex, condition (FOODTRAY vs. OTHER), and sessions, the last being repeated measures within subjects. Rate measures were log-transformed and percentages were 2 - arcsine [square root (x)] transformed (Winer, 1962).

Figure 1 shows tray-entry rates during the autoshaping sessions. Subjects previously exposed to the FOODTRAY condition made more tray-entry responses during autoshaping than did subjects exposed to the OTHER condition $[F(1,28)=35.00, p<.01]$. Tray-entry rates decreased over sessions for all subjects $[F(4,112)=$ $32.76, p<.01]$, but decreased faster for males than for females [sex $\times$ sessions, $F(4,112)=9.01, p<.01$ ] Tray-entry rates also decreased faster for subjects exposed to the FOODTRAY condition than for subjects exposed to the OTHER condition during preexposure [condition $\times$ sessions, $F(4,112)=23.95, p<.01]$. No other significant main or interaction effects were observed.

Figure 2 shows the percentage of CS presentations with at least one CS contact response. The subjects that were previously exposed to the FOODTRAY condition seemed less likely to contact the CS during its presentation than did the subjects exposed to the OTHER condition, although this effect was only marginally significant 


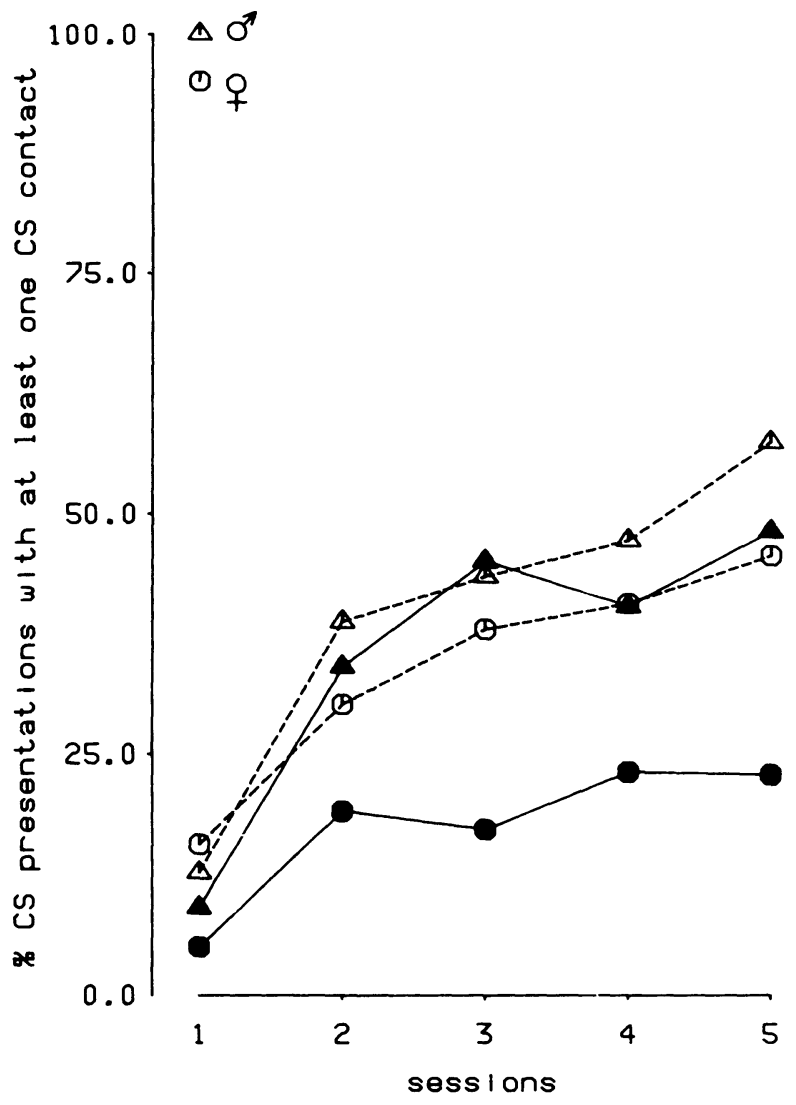

Figure 2. Percentage of CS presentations in which at least one CS contact occurred for groups of male (triangles) and female (circles) Wistar rats that were exposed to either FOODTRAY (solid lines) or OTHER (dotted lines) reinforcement contingencies during preexposure to unsignaled food presentations.

$[F(1,28)=3.50, p<.072]$. The percentage of trials with at least one CS contact increased over sessions $[F(4,112)$ $=17.83, p<.01]$. Males contacted the CS on more trials than did females $[F(1,28)=4.22, p<.05]$, irrespective of the previously experienced preexposure conditions [sex $\times$ condition, $F(1,28)<1.0$, n.s.].

CS contact rates are depicted in Figure 3 . The subjects that were previously exposed to the OTHER condition exhibited higher CS contact rates than did the subjects that were exposed to the FOODTRAY condition during preexposure $[F(1,28)=4.64, p<.05]$. Contact rates increased over sessions $[F(4,112)=16.76, p<.01]$. Males contacted the CS more frequently than did females $[F(1,28)=4.68, p<.05]$, but this effect was again independent of the experienced contingencies during preexposure [sex $\times$ condition, $F(1,28)<1.0$, n.s.].

\section{DISCUSSION}

The results of the present experiment show that differential conditioning of food-tray directed behavior during preexposure affects the subsequent acquisition of stimulus-directed behavior in autoshaping procedures. The subjects that were conditioned to engage in low levels of food-tray directed behavior during preexposure engaged more in stimulus-directed behavior during autoshaping than did the subjects trained to engage in high levels of food-tray directed behavior. These results suggest that the retarded acquisition of autoshaped responding following preexposure to response-independent food (Balsam \& Schwartz, 1981; Downing \& Neuringer, 1976; Engberg et al., 1972; Timberlake, 1986; Tomie, 1981) may be a direct function of the extent to which subjects engage in food-tray directed behavior during preexposure.

It has been argued that subjects that are preexposed to unsignaled food presentation engage less frequently in stimulus-directed behavior because stimuli other than the autoshaping stimulus have become reliable predictors of food presentation (context-blocking hypothesis) (e.g., Balsam, Locurto, Terrace, \& Gibbon, 1980; Tomie, 1981). The contextblocking hypothesis states that subjects direct their behavior toward the most salient stimulus predictive of food presentation in the absence of a stimulus that signals the imminent presentation of food. When subjects are subsequently exposed to food presentation that is preceded by the presentation of a stimulus, conditioning to the stimulus is blocked, either because the stimulus is redundant with respect to the delivery of food, or because other environmental cues have exhausted all associative strength connected to the presentation of food (Kamin, 1969; Mackintosh, 1975; Rescorla \& Wagner, 1972). Other investigators, however, have suggested that preexposure to unsignaled food presentation affects subsequent stimulus-directed behavior in autoshaping because behavior incompatible with stimulus-directed behavior has been conditioned (Schwartz, Reisberg, \& Vollmecke, 1974).

The results of the present experiment support a context-blocking explanation of preexposure effects on the acquisition of stimulus-directed responding in autoshaping procedures (Balsam et al., 1980; Timberlake, 1986; Tomie, 1981). It may be that during preexposure to unsignaled, response-independent food, stimuli associated with the food tray

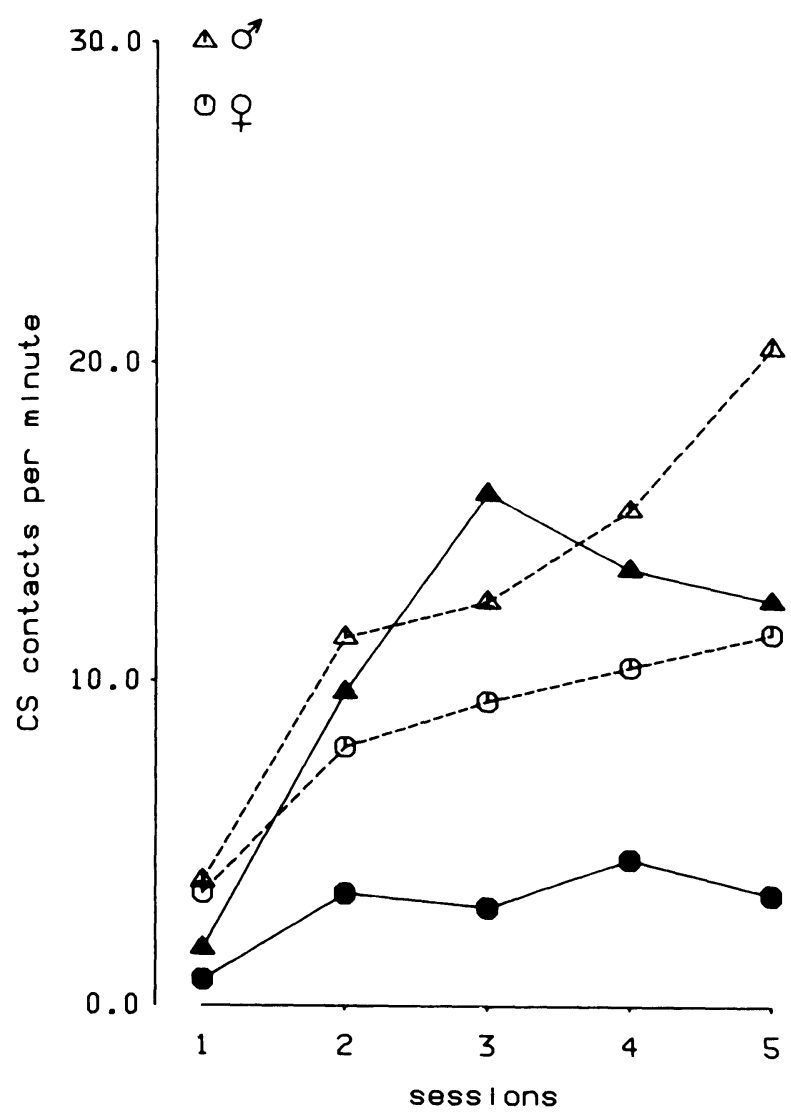

Figure 3. CS contact rates (leverpresses per minute) for groups of male (triangles) and female (circles) Wistar rats that were exposed to either FOODTRAY (solid lines) or OTHER (dotted lines) reinforcement contingencies during preexposure to unsignaled food presentations. 
become the most salient and predictive stimuli with respect to food delivery. Stimulus-directed behavior should thus be more retarded when foodtray-directed behavior has been explicitly conditioned during preexposure than when such behavior has been explicitly not conditioned. The results of the present experiment support such an analysis. A number of other experiments, most notably those in which preexposure and autoshaping sessions occurred in different environments, have provided additional evidence in favor of the context-blocking hypothesis by showing that context-blocking effects were observed only when preexposure and autoshaping occurred in the same physical environment (Tomie, 1976a, 1976b, 1981; Tomie, Murphy, Fath, and Jackson, 1980).

A response-competition paradigm, on the other hand, seems less parsimonious than a context-blocking theory. Given the differential contingencies of the two preexposure groups in the present study, the response-competition hypothesis would not predict any obvious differences between the groups in the acquisition rate of stimulus-directed behavior during autoshaping, unless it is assumed that some responses compete more with stimulus-directed activity than do others. However, under this assumption, one would have expected the FOODTRAY group to show higher levels of stimulus-directed behavior than the OTHER group, because stimulus-directed activities during autoshaping have frequently been interpreted to result from "stimulus substitution" processes (see Schwartz \& Gamzu, 1977).

The results of the present experiment confirm and extend the findings on behavioral differences between the sexes observed in other experimental procedures. Males exhibited higher levels of lever-directed behavior than did females, both when food presentation was contingent upon leverpressing and in autoshaping procedures when the lever was a signal for the upcoming availability of food (Heinsbroek, van Haaren, Zantvoord, \& van de Poll, 1987; van Haaren et al., 1987; van Hest, van Haaren, \& van de Poll, 1987a, 1987b; van Hest \& van Haaren, 1989). The results of the present experiment show that preexposure to unsignaled food presentations did not differentially affect the behavior of males and females in an autoshaping procedure.

\section{REFERENCES}

Balsam, P. D., Locurto, C. M., Terrace, H. S., \& Gibbon, J. (1980). A search for preexposure effects in autoshaping: Effects of US-only or random CS-US presentations, intertrial interval duration, and number of pretraining trials. Psychological Records, 30, 561-570.

Balsam, P. D., \& SCHWARTZ, A. (1981). Rapid contextual conditioning in autoshaping. Journal of Experimental Psychology: Animal Behavior Processes, 1, 382-393.

BoAKEs, R. A. (1977). Performance on learning to associate a stimulus with positive reinforcement. In H. Davis \& H. M. B. Hurwitz (Eds.), Operant-Pavlovian interactions (pp. 67-97). Hillsdale, NJ: Erlbaum.

Downing, K., \& NeUringer, A. (1976). Autoshaping as a function of prior food presentations. Journal of the Experimental Analysis of Behavior, 26, 463-469.

Engberg, L. A., Hansen, G., Welker, R. L., \& Thomas, D. R. (1972). Acquisition of key-pecking via auto-shaping as a function of prior experience: “Learned laziness?”' Science, 178, 1002-1004.

Heinsbroek, R. P. W., van HaARen, F., Zantvoord, F., \& VAN DE Poll, N. E. (1987). Sex differences in response rates during random ratio acquisition: Effects of gonadectomy. Physiology \& Behavior, 39, 269-272.

InNis, N. K., Simmelhag-Grant, V. L., \& Staddon, J. E. R. (1983). Behavior induced by periodic food delivery: The effects of interfood interval. Journal of the Experimental Analysis of Behavior, 39, 309-322.
KAMIN, L. J. (1969). Predictability, surprise, attention and conditioning. In B. A. Campbell \& R. M. Church (Eds.), Punishment and aversive behavior (pp. 279-296). New York: Appleton-Century-Crofts.

Mackintosh, N. J. (1975). A theory of attention: Variations in the associability of stimuli with reinforcement. Psychological Review, 82, 276-298.

Rescorla, R. A., \& Wagner, A. R. (1972). A theory of Pavlovian conditioning: Variations in the effectiveness of reinforcement and nonreinforcement. In A. H. Black \& W. F. Prokasy (Eds.), Classical conditioning: Vol. 2. Current theory and research (pp. 64-99). New York: Appleton-Century-Crofts.

Schwartz, B., \& GamzU, E. (1977). Pavolovian control of operant behavior. In W. K. Honig \& J. E. R. Staddon (Eds.), Handbook of operant behavior (pp. 53-97). Englewood Cliffs, NJ: Prentice Hall.

Schwartz, B., ReISBERG, D., \& Vollmecke, T. (1974). Effects of treadle training on autoshaped keypecking: Learned laziness and learned industriousness or response competition? Bulletin of the Psychonomic Society, 3, 369-372.

TIMBERLAKE, W. (1986). Unpredicted food produces a mode of behavior that affects rats' subsequent reactions to a conditioned stimulus: A behavior-system approach to "context blocking." Animal Learning \& Behavior, 14, 276-286.

Tomie, A. (1976a). Interference with autoshaping by prior context conditioning. Journal of Experimental Psychology: Animal Behavior Processes, 2, 323-334.

TомIE, A. (1976b). Retardation of autoshaping: Control by contextual stimuli. Science, 192, 1244-1246.

TомiE, A. (1981). Effect of unpredictable food on the subsequent acquisition of autoshaping: Analysis of the context-blocking hypothesis. In C. M. Locurto, H. S. Terrace, \& J. Gibbon (Eds.), Autoshaping and conditioning theory (pp. 181-215). New York: Academic Press.

Tomie, A., MurPhy, A. L., FATH, S., \& JACKSON, R. L. (1980). Retardation of autoshaping following pretraining with unpredictable food: Effects of changing the context between training and testing. Learning \& Motivation, 11, 117-134.

van HaAren, F., van Hest, A., \& Van de Poll, N. E. (1987). Acquisition and reversal of a discriminated autoshaped response in male and female rats: Effects of long or short and fixed or variable intertrial interval durations. Learning \& Motivation, 18, 220-233.

van Hest, A., van der Schoot, F., Kop, P., \& van HaAren, F. (1987). Dissociation of instrumental and Pavlovian contingencies in a discriminated instrumental procedure. Behavioural Processes, 15, 249-258.

van Hest, A., \& VAN HAaren, F. (1989). The effects of gonadectomy and chronic testosterone suppletion on the autoshaped response of male and female Wistar rats. Bulletin of the Psychonomic Society, 27, 45-48. van Hest, A., van HaAren, F., Kop, P., \& Van der Schoot, F. (1986). Stimulus- and feeder-directed behavior in a long-box: Effect of fixed versus variable time schedules of food presentation. Animal Learning \& Behavior, 14, 168-172.

van Hest, A., van HaAren, F., \& Van de Poll, N. E. (1987a). Behavioral differences between male and female Wistar rats on DRL schedules: Effect of stimuli promoting collateral activities. Physiology \& Behavior, 39, 255-261.

van Hest, A., van HaAren, F., \& van de Poll, N. E. (1987b). Behavioral differences between male and female Wistar rats in food rewarded lever holding. Physiology \& Behavior, 39, 263-267.

WINER, B. J. (1962). Statistical principles in experimental design. New York: McGraw-Hill.

(Manuscript received January 2, 1989). 